\title{
The Effect of Steel Leveler Parameters on Vibration Features
}

\author{
Riku-Pekka Nikula ${ }^{1} \quad$ Konsta Karioja $^{2}$ \\ ${ }^{1}$ Control Engineering, University of Oulu, Finland, riku-pekka.nikula@oulu.fi \\ ${ }^{2}$ Mechatronics and Machine Diagnostics, University of Oulu, Finland, konsta.karioja@oulu.fi
}

\begin{abstract}
The development of steel products with various characteristics increases the need for timely and preventive maintenance and condition monitoring of the production machinery. For instance, the roller levelers at modern steel factories are exposed to a high variation of forces due to the large range of steels leveled. In this study, the vibration measured from a steel leveler used for cold steel strips is analyzed with the goal to identify the effects different operational conditions have. Features such as generalized norms, generalized norm sums and the crest factor are computed from the vibration signals. The effects of the steel strip properties and the operational parameters of the machine on these features are then analyzed. The obtained information can be utilized in the models that are used as planning tools for the preventive maintenance of the steel leveler.
\end{abstract}

Keywords: feature extraction, roller leveling, signal processing, vibration

\section{Introduction}

The machines and devices in the modern steel industry are exposed to a high variation of load. This is a common characteristic especially at the factories which produce special steels. The special steel strips have properties such as exceptionally high yield strength. Major stresses are therefore inflicted on the processing equipment such as roller levelers during the production. This increases the risks of damage. The major forces make the roller leveler behave in an undesirable manner which makes, e.g., the mechanical load limiters break and the work rollers slip. Other common detriments encountered in the steel levelers include bearing damage in the rollers and the breakage and abrasion of the work rollers. These factors may consequently weaken the quality of the final steel product or cause a notable production loss due to the maintenance time. Therefore, the real-time monitoring of machine condition and the prediction of the effects of a specific steel product on the leveler are important subjects of research.

In this paper, the effects of the operating conditions during the steel leveling are studied from the vibration signal measured from the bodywork of a steel leveler. The studied roller leveler is used for strips of cold steel in a sheet line. The sheets are cut from the strip next to the leveler using a flying shear. The cutting causes impacts which are conducted to the leveler and the measured signal. Figure 1 depicts two examples of the measured signal during the processing of steel strips in the steel leveler. Figure 1 indicates that the effects of the steel strip properties and the operational parameters of the leveler are divergent in these cases. Differences between the leveling events can be commonly seen in the general signal level, in the impact magnitude of the sheet cutting and in the duration of the leveling events.

The identification of changes in the behavior of the monitored system can be done by using features (Lahdelma and Juuso, 2011). In this study, derivatives are first calculated in order to magnify the effects appearing in the signal. This is done according to the definitions by (Lahdelma, 1997) for real order derivatives. The actual vibration features are then extracted from the signal using the generalized norms introduced by (Lahdelma and Juuso, 2008). These norms are also used as the basis for other features such as generalized norm sums and the crest factor.

The signal derivatives and generalized norms have been previously used to demonstrate that different steel grades inflict different stress levels on a steel leveler (Karioja et al, 2015). Features based on the generalized norms have been proposed also as stress indicators for the same application (Nikula et al, 2017). In contrast to these studies, this paper addresses the effects of the operational parameters of the machine on the measured vibration. The effects of the steel strip properties on the general signal level and on the relative peak magnitude are studied as well. The correlations of the signal features with the machine parameters and steel strip properties are studied using Pearson's correlation coefficient. This information is useful for the development of data-driven models that are used in the preventive maintenance of the roller levelers.

\section{Materials and Methods}

\subsection{Steel Leveler}

The purpose of roller leveling is the elimination of various shape defects in the material. Steel coils contain flatness defects caused by uneven stresses and defects resulting from thickness variation across the product width (Smith, 1997). The stress patterns create 
transverse and longitudinal curvature. Center and edge waves are caused by difference in the length of sheet between the center and the edges (Park and Hwang, 2002). Roller leveling is done by subjecting the strip to multiple back and forth bending sequences with decreasing roll penetrations as illustrated in Figure 2. The principle of roller leveling is based on controlling the plastic deformation through the thickness of the material. The plastic deformation determines the resultant flatness and memory and it also affects the required force. The roll force is a function of material thickness, width, yield strength, roll spacing and the extent of plastic deformation (Smith, 1997). A proper combination of the operational parameters is therefore needed for the required leveling result.

A schematic representation of a roller leveler is shown in Figure 2. The leveler under investigation is a four-high leveling machine used for strips of cold steel in a sheet line. The sheet cutting is performed simultaneously with the leveling without a need to stop the strip in the leveler due to the cutting. The cutting causes impacts that are conducted to the leveler and emerge as peaks in the monitored vibration signal which is also demonstrated in the lower graph in Figure 1.

\subsection{Steel Leveler Parameters}

The studied operational parameters of the leveler are presented in Table 1. The leveling of one complete steel strip is considered here as a leveling event. Exact time stamps for the operational parameters were unavailable and therefore the medians of each parameter represent the whole leveling event. The median represents the general level of an operational parameter during the leveling event. To precisely identify the instantaneous effect of an operational parameter on the vibration signal, the exact synchronization of time stamps is required.

The studied steel strip properties include yield strength, strip length, strip weight, strip width and strip thickness. The properties of the studied steel strips varied extensively. The range of the yield strength was approximately 200-1600 MPa; the length range was 68$1161 \mathrm{~m}$; the thickness range was $1.98-15.21 \mathrm{~mm}$; and the number of cut sheets was 4-465. The vibration signals from the leveling of altogether 739 steel strips were analyzed. The most common steel grade from 53 steel grades was a cold formable steel grade with yield strength around $400 \mathrm{MPa}$. This steel grade was leveled 123 times.

\subsection{Vibration Measurements}

The measurements were done at the SSAB rolling mill in Raahe, Finland. The accelerometer was studmounted in the middle of the runway on the bodywork supporting the lower supporting rolls. The acceleration was measured horizontally in the cross direction relative to the direction of the roller track. The used accelerometer was SKF CMSS 787A-M8, which has the frequency response from $0.7 \mathrm{~Hz}$ to $10 \mathrm{kHz}$ with $\pm 3 \mathrm{~dB}$ deviation. The measurement hardware included NI 9234 data acquisition card and NI CompactRIO for the data recording. The sampling rate was $25.6 \mathrm{kHz}$ and the only filter used in hardware level was the built-in antialiasing filter of the data acquisition card. The measurement system was calibrated after the completion of measurements using a hand-held calibrator.

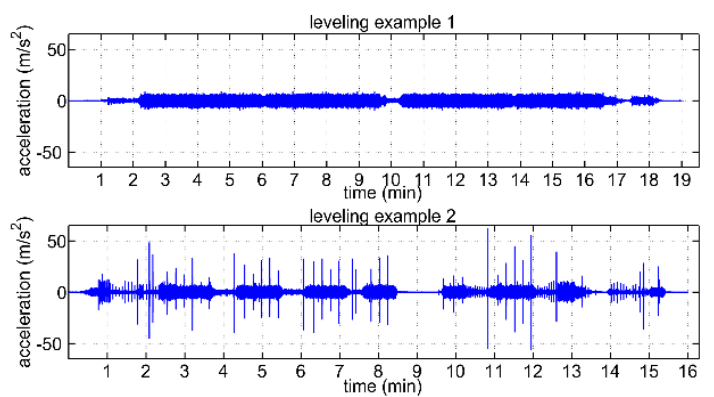

Figure 1. The acceleration signals from the leveling of two steel strips.

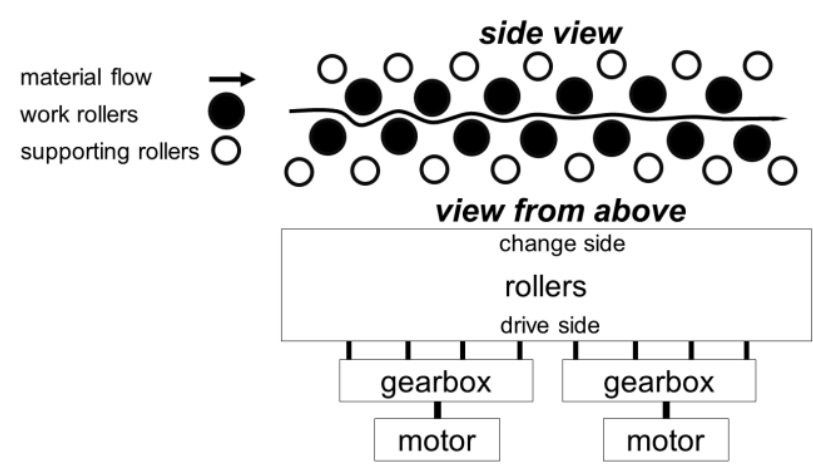

Figure 2. A schematic view of a roller leveler.

Table 1. The Studied Operational Parameters.

\begin{tabular}{|l|l|l|}
\hline operational parameter & $\begin{array}{l}\text { parameter } \\
\text { identifier }\end{array}$ & measure \\
\hline exit gap, drive side & $P 1$ & $\mathrm{~mm}$ \\
\hline exit gap, difference between sides & $P 2$ & $\mathrm{~mm}$ \\
\hline exit gap, change side & $P 3$ & $\mathrm{~mm}$ \\
\hline degree of plastic deformation & $P 4$ & $\%$ \\
\hline $\begin{array}{l}\text { entrance gap, drive side } \\
\text { entrance gap, difference between } \\
\text { sides }\end{array}$ & $P 6$ & $\mathrm{~mm}$ \\
\hline $\begin{array}{l}\text { entrance gap, change side } \\
\text { drawing force caused by roller } \\
\text { leveler }\end{array}$ & $P 7$ & $\mathrm{~mm}$ \\
\hline $\begin{array}{l}\text { drawing force caused by entry } \\
\text { pinch roller }\end{array}$ & $P 9$ & $\mathrm{kN}$ \\
\hline
\end{tabular}




\subsection{Signal Processing}

The signals were processed using computational methods which are integration, derivation and filtering. The processing was done in the frequency domain by manipulating the sequence of complex numbers resulting from fast Fourier transform (FFT). Lahdelma (Lahdelma, 1997) defined real order derivative $x^{(\alpha)}$ of the function $x(t)=X e^{i \omega t}$ in the form

$$
x^{(\alpha)}=\omega^{\alpha} X e^{i\left(\omega t+\alpha \frac{\pi}{2}\right)},
$$

where $\alpha$ is the order of derivative, $\omega$ is angular frequency, $X$ is amplitude, $e$ is the Napierian number, $i$ is the imaginary unit and $t$ is the time variable. The derivative with respect to time can be calculated by multiplying every term $X_{k}$ of the FFT by $\left(i \omega_{k}\right)^{\alpha}$ and then using the inverse of FFT. The integrals can be calculated similarly by using negative values of $\alpha$. Furthermore, the value is not limited to integers but any real or complex number can be used (Lahdelma, 1997; Lahdelma and Kotila, 2005). Signals are filtered by multiplying the unwanted frequency components by zero.

In this study, the order of derivation was $\alpha=\{1,2,3$, 4 . The variable $x^{(2)}$ corresponds to acceleration and $x$ stands for displacement. The velocity signal $(\alpha=1)$ was filtered so that it included the frequencies $2-1000 \mathrm{~Hz}$. The other signals were filtered only by the antialiasing filter embedded in the data acquisition card. The calculation of derivatives was done only for the leveling events that took less than 60 minutes. The events with longer duration were removed. Otherwise, the preprocessing was done according to the procedure presented in (Nikula et al, 2017). Five per cent of data was removed from the start and the end of each event to remove the effect of windowing in signal processing.

\subsection{Vibration Features}

The generalized norm is defined by

$$
\left\|X^{(\alpha)}\right\|_{p}=\left[\frac{1}{N} \sum_{i=1}^{N}\left|x_{i}^{(\alpha)}\right|^{p}\right]^{\frac{1}{p}} .
$$

This feature is known as the $l_{p}$ norm of signal $x^{(\alpha)}$ where $p$ is the order of the norm, $\alpha$ is the order of derivation and $N$ is the number of signal values. The $l_{p}$ norm has the same form as the generalized mean which is also known as the Hölder mean or power mean (Bullen, 2003). The root mean square and the peak value are special cases of the norm (2) when $p=2$ and $p=\infty$, respectively. The large order of the norm magnifies the effect of the peaks, whereas the small order of the norm diminishes them.

The rms $\left(l_{2}\right)$ was used to study the effect of the machine parameters and strip properties on the general signal level in this study. The crest factor $(C)$ is the ratio between the absolute peak value $\left(l_{\infty}\right)$ and $\mathrm{rms}\left(l_{2}\right)$. The crest factor was used to study the relative magnitude of the impacts seen in the signal. These two features were computed from the segments, which had the duration of ten seconds. The mean of segments from the complete event was then used in the analysis of the effects.

The sums of $l_{0.1}, l_{2}, l_{4}, l_{10}$, and $l_{1}+l_{10}$ were used to study the effects of the operational parameters of the machine only. A study concerning the correlations between the steel strip properties and the generalized norm sums is presented in (Nikula et al, 2017). The generalized norms were calculated using one-second samples in this case. Each sum was divided by the mean of the corresponding sums from the events, during which the most common steel grade was processed. Therefore, the value 1 corresponds to the mean of the most common steel grade in each sum. This operation was done in order to make the summation of $l_{1}$ and $l_{10}$ practical.

\subsection{Pearson's Correlation Coefficient}

The correlation coefficient, $R_{x y}$, is defined by

$$
R_{x y}=\frac{\sum_{i=1}^{n}\left(x_{i}-\bar{x}\right)\left(y_{i}-\bar{y}\right)}{\sqrt{\sum_{i=1}^{n}\left(x_{i}-\bar{x}\right)^{2} \sum_{i=1}^{n}\left(y_{i}-\bar{y}\right)^{2}}},
$$

where $x_{i}$ and $y_{i}$ are two different variables; $\bar{x}$ and $\bar{y}$ are their corresponding sample means; and $n$ is the total number of observations. $R_{x y}$ close to \pm 1 indicates strong linear correlation whereas $R_{x y}$ close to 0 indicates weak linear correlation between the variables.

\section{Results and Discussion}

\subsection{Correlations of Operational Conditions}

Table 2 shows the correlation coefficients between the operational parameters of the machine and the steel strip properties based on the 739 events studied. The yield strength had a rather strong negative correlation with the gap values $(P 1, P 3, P 5$, and $P 7)$, a strong negative correlation with the plastic deformation $(P 4)$ and a strong positive correlation with the drawing force caused by the machine $(P 8)$. The strip length had rather similar relationships with these operational parameters. The negative correlations with gap values were even stronger, but the correlations with the plastic deformation and the drawing force caused by the machine were slightly weaker. The correlations of strip weight and width with the operational parameters were generally weaker. The strong correlation of the strip width with the drawing force caused by the entry pinch roller $(P 9)$ is an exception. The correlations of the strip thickness were the opposite to the correlations of the yield strength or the length with the same operational parameters. This means that the thickness has a strong positive correlation with the gap values and the plastic deformation but quite strong negative correlation with the drawing force caused by the machine.

Table 2 roughly illustrates how the properties of the steel strips influence the operation of the roller leveler. In practice, the operational parameters are set based on the steel properties and then manipulated during the 
leveling by the operator in the steelworks. Moreover, the strong correlations in Table 2 indicate that the medians of the operational parameters represent the operation sufficiently for the analysis purpose.

\subsection{Correlations of Steel Strip Properties with Vibration Features}

Table 3 shows the correlation coefficients of $\mathrm{rms}$ and crest factor with the steel strip properties. The rms calculated from the acceleration signal $x^{(2)}$ correlated the strongest with the yield strength, whereas the other steel strip properties had weak correlation. The rms values from the higher order derivatives of the signal indicated similar behavior. In the case of the velocity signal $x^{(1)}$, the correlation with the yield strength was slightly lower. The results indicate that the general signal level correlates the strongest with the yield strength considering the studied steel strip properties.

Figure 3 shows the rms of the $x^{(2)}$ signal together with two steel strip properties that had the strongest correlation. This 3D scatter plot illustrates that the strips with high yield strength are relatively thin. The thickness seems to have a positive correlation with rms if the yield strength is fixed although the general correlation is negative as shown in Table 3 .

The crest factor had negative correlation with the strip length and positive correlation with the thickness according to Table 3. This result indicates that a thick steel strip results in relatively large impacts appearing in the signal. These impacts are mostly resulting from the sheet cutting using the flying shear. The weight and the width of the strip had low correlations in general. The effect of the yield strength varied based on the order of derivation. When the strip is thick, the yield strength and the strip length are relatively low, which explains the negative correlations of these two variables with the crest factor. When the velocity signal was used, the effect of weak impacts reduced on the crest factor. On the other hand, the strong impacts clearly stood out. This effect is shown in Figure 4. When the other signal derivatives were used, also the weaker impacts were magnified. This behavior is illustrated in the lower part of Figure 4. The level of the crest factor was lower in the case of $x^{(1)}$ signal compared with the higher order derivatives of the signal.

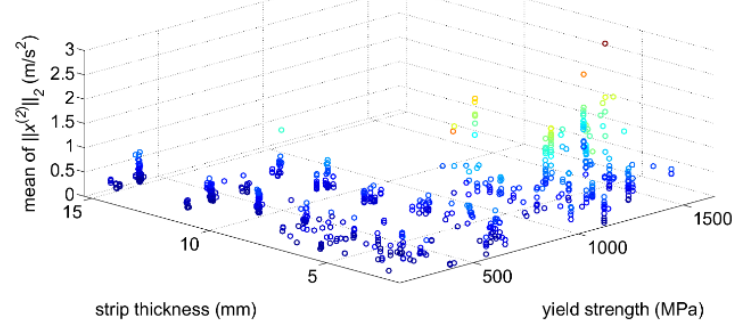

Figure 3. The effect of yield strength and thickness on the rms calculated from the acceleration signals.

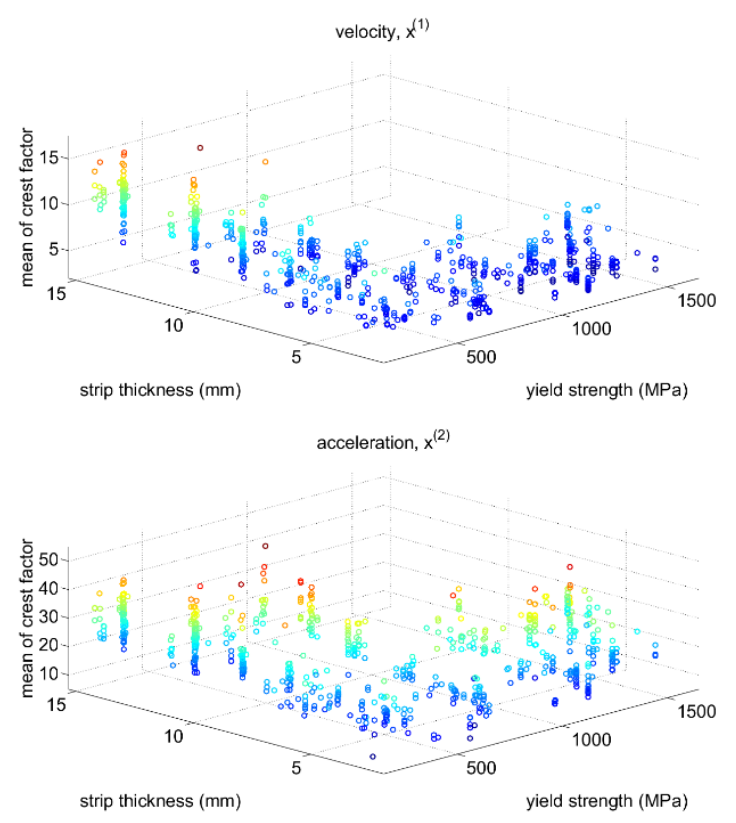

Figure 4. The effect of yield strength and thickness on the crest factor using $x^{(1)}$ and $x^{(2)}$ signals.

Table 2. Correlations between the Operational Parameters and the Steel Strip Properties.

\begin{tabular}{|l|l|l|l|l|l|}
\hline id. & $\begin{array}{l}\text { yield } \\
\text { strength }\end{array}$ & length & weight & width & thickness \\
\hline$P 1$ & -0.656 & -0.793 & 0.441 & 0.277 & 0.991 \\
\hline$P 2$ & 0.218 & -0.075 & 0.070 & 0.093 & -0.017 \\
\hline$P 3$ & -0.662 & -0.790 & 0.438 & 0.274 & 0.990 \\
\hline$P 4$ & -0.875 & -0.630 & 0.401 & 0.286 & 0.758 \\
\hline$P 5$ & -0.673 & -0.810 & 0.490 & 0.332 & 0.973 \\
\hline$P 6$ & -0.036 & -0.209 & 0.151 & 0.159 & 0.153 \\
\hline$P 7$ & -0.676 & -0.809 & 0.488 & 0.329 & 0.973 \\
\hline$P 8$ & 0.853 & 0.510 & -0.312 & -0.183 & -0.683 \\
\hline$P 9$ & -0.116 & -0.201 & 0.561 & 0.931 & 0.156 \\
\hline
\end{tabular}

Table 3. Correlations of the Steel Strip Properties with rms and Crest Factor.

\begin{tabular}{|l|l|l|l|l|l|}
\hline feature & $\begin{array}{l}\text { yield } \\
\text { strength }\end{array}$ & length & weight & width & $\begin{array}{l}\text { thick- } \\
\text { ness }\end{array}$ \\
\hline$\left\|x^{(1)}\right\|_{2}$ & 0.487 & 0.048 & 0.094 & 0.039 & -0.085 \\
\hline$\left\|x^{(2)}\right\|_{2}$ & 0.660 & 0.192 & 0.072 & 0.056 & -0.310 \\
\hline$\left\|x^{(3)}\right\|_{2}$ & 0.630 & 0.207 & 0.031 & 0.021 & -0.328 \\
\hline$\left\|x^{(4)}\right\|_{2}$ & 0.634 & 0.217 & 0.022 & 0.013 & -0.339 \\
\hline$C, x^{(1)}$ & -0.516 & -0.569 & 0.275 & 0.175 & 0.759 \\
\hline$C, x^{(2)}$ & 0.091 & -0.401 & 0.248 & 0.099 & 0.384 \\
\hline$C, x^{(3)}$ & -0.217 & -0.422 & 0.179 & 0.126 & 0.470 \\
\hline$C, x^{(4)}$ & -0.251 & -0.423 & 0.185 & 0.154 & 0.468 \\
\hline
\end{tabular}




\subsection{Correlations of Operational Parameters with Vibration Features}

Table 4 shows the correlation coefficients of rms and crest factor with the operational parameters of the machine. The correlations of rms are shown using only $x^{(1)}$ and $x^{(2)}$ signals. The rms of $x^{(3)}$ and $x^{(4)}$ signals had almost the same correlations as the rms of $x^{(2)}$ signal. In general, the linear correlations between the operational parameters and rms were rather low. The drawing force caused by the leveler $(P 8)$ had the strongest correlation. As shown in Table 2, this parameter also had a strong correlation with the yield strength, which has a large influence on the rms level as shown in the previous Section.

The crest factor had a strong positive correlation with the roller gap values and the degree of plastic deformation when calculated from the $x^{(1)}$ signal. These correlations were weaker using the higher order derivatives of the signal. The drawing force caused by the roller leveler $(P 8)$ had a stronger negative correlation with the crest factor of $x^{(1)}$ signal compared with the crest factor of the other signals. The correlations between the yield strength and the crest factor of different signal derivatives indicated a similar behavior which is shown in Table 3.

Table 5 shows the correlations between the operational parameters of the machine and the generalized norm sums calculated from the $x^{(2)}$ signal. The generalized norm sums correlated the strongest with the drawing force caused by the roller leveler. The gap values and the degree of plastic deformation had substantially strong negative correlations. The results indicate that the correlations were the highest when the order of the norm was the lowest in these cases. In most cases, the second highest correlation was observed with the norm combination $l_{1}+l_{10}$.

The effects of operational parameters $P 1, P 4, P 5$, and $P 8$ on $l_{0.1}$ sum are depicted in Figure 5. According to the presented gap values, a thick steel strip causes a low accumulation of norm values. When the gap is reduced, the norm sums increase and an increasing variation in the sums is observed. This effect is seen in the graphs illustrating the effects of gap values and the degree of plastic deformation. The norm sums seem to increase together with the drawing force caused by the roller leveler. When the operational parameters are considered fixed, the variation in the norm sums can be explained by the effects of other variables. After all, the effects seen in the vibration are the result of the behavior of a multivariate system.

\subsection{Discussion}

The effects of the operational parameters of the steel leveler on the vibration signals were demonstrated in this study. Moreover, the results indicated that the operational parameters have strong correlations with the different steel strip properties. This implies that the characteristics of the vibration could be predicted with an approximate precision before the leveling is done based solely on the steel strip properties.

The results indicated that the steel strips with high yield strength inflict relatively high vibration level, which is seen in the rms values. According to the results in Table 4, the drawing force caused by the leveler apparently had an effect on this as well. Figure 3 also indicates that the steel thickness affects the rms value when the yield strength is kept fixed. This implies that the strip thickness could be used to predict the rms level during the processing of a specific steel grade because the yield strength is fixed in that case. The high level of rms may be an indication of the potential risks of slipping in the work rollers or abrasion, for instance.

The impacts caused by the sheet cutting manifested themselves in the crest factor especially with thick strips as shown in Figure 4. In addition, other variables such as the yield strength had an influence especially in the case of relatively thin strips. The roller gap values indicated a positive correlation with the crest factor as well. The impacts presumably stress the bearings of the work rollers and supporting rollers, which are under a major load during the leveling.

The roller gap values had quite strong negative correlation with the generalized norm sums. This indicates that the thin strips, which are also long, accumulate higher sums compared with the thick strips. This means that the leveler is stressed a relatively long duration. The generalized norm sums could be used for the general machine stress evaluation.

The roller gap differences between the sides ( $P 2$ and $P 6)$ had low correlations with the vibration features. These parameters had low correlations with the studied steel strip properties as well. The entry pinch roller $(P 9)$ had weak correlations with the vibration features, but a strong correlation with the strip width. The strip weight and the width had low correlations with the studied vibration features as well. These parameters apparently have poor applicability to the prediction of the studied effects appearing in the vibration signal. The effects of the positions of the supporting rollers were rejected in this study because information on the possible steel strip shape flaws was unavailable. In general, the supporting rollers are adjusted to correct flatness defects (Smith, 1997).

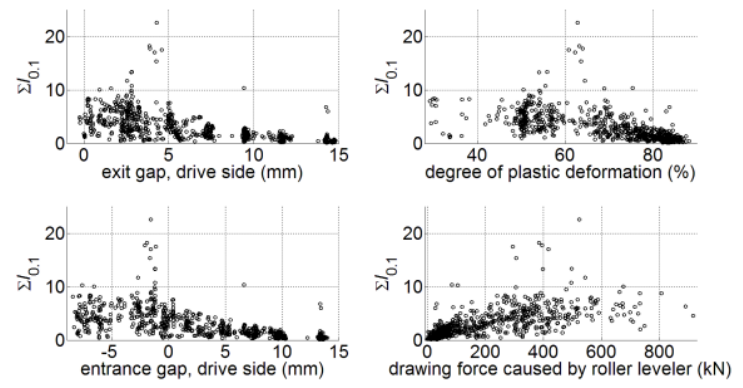

Figure 5. The effect of four operational parameters on $l_{0.1}$ sums using $x^{(2)}$ signals. 
Table 4. Correlations of the Operational Parameters with rms and Crest Factor.

\begin{tabular}{|l|l|l|l|l|l|l|}
\hline & $x^{(1)}$ & $x^{(2)}$ & $x^{(1)}$ & $x^{(2)}$ & $x^{(3)}$ & $x^{(4)}$ \\
\hline id. & \multicolumn{2}{|l|}{$r m s$} & \multicolumn{4}{l|}{ Crest Factor } \\
\hline$P 1$ & -0.135 & -0.366 & 0.760 & 0.344 & 0.457 & 0.459 \\
\hline$P 2$ & 0.154 & 0.175 & -0.075 & 0.222 & 0.008 & -0.014 \\
\hline$P 3$ & -0.139 & -0.371 & 0.762 & 0.338 & 0.456 & 0.459 \\
\hline$P 4$ & -0.280 & -0.469 & 0.609 & 0.128 & 0.320 & 0.342 \\
\hline$P 5$ & -0.149 & -0.350 & 0.734 & 0.365 & 0.467 & 0.470 \\
\hline$P 6$ & 0.011 & 0.005 & 0.057 & 0.230 & 0.085 & 0.073 \\
\hline$P 7$ & -0.150 & -0.352 & 0.736 & 0.361 & 0.467 & 0.471 \\
\hline$P 8$ & 0.434 & 0.588 & -0.522 & -0.088 & -0.289 & -0.315 \\
\hline$P 9$ & 0.059 & 0.087 & 0.115 & 0.059 & 0.072 & 0.091 \\
\hline
\end{tabular}

Table 5. Correlations of the Operational Parameters with the Sums of the Generalized Norms.

\begin{tabular}{|l|l|l|l|l|l|}
\hline$i d$. & $\sum l_{0.1}$ & $\sum l_{2}$ & $\sum l_{4}$ & $\sum l_{10}$ & $\sum\left(l_{1}+l_{10}\right)$ \\
\hline$P 1$ & -0.593 & -0.511 & -0.483 & -0.484 & -0.532 \\
\hline$P 2$ & 0.108 & 0.141 & 0.160 & 0.168 & 0.145 \\
\hline$P 3$ & -0.595 & -0.515 & -0.487 & -0.488 & -0.536 \\
\hline$P 4$ & -0.622 & -0.577 & -0.561 & -0.560 & -0.593 \\
\hline$P 5$ & -0.600 & -0.507 & -0.473 & -0.472 & -0.528 \\
\hline$P 6$ & -0.080 & -0.050 & -0.026 & -0.013 & -0.044 \\
\hline$P 7$ & -0.601 & -0.508 & -0.475 & -0.474 & -0.529 \\
\hline$P 8$ & 0.658 & 0.618 & 0.592 & 0.582 & 0.626 \\
\hline$P 9$ & 0.002 & 0.051 & 0.061 & 0.057 & 0.041 \\
\hline
\end{tabular}

\section{Conclusions}

The effects of steel leveler parameters on vibration features were studied based on the observed linear correlations. Signal derivatives were calculated to magnify the effects. Crest factor, rms and generalized norm sums were used as the vibration features. The crest factor, which shows the ratio of the peak amplitude and rms, had strong correlation especially with strip thickness and the roller gap parameters. The general magnitude of a signal, defined by rms, had the strongest correlations with the yield strength of strip and the drawing force caused by the roller leveler. The generalized norm sums, which can be used to indicate stress accumulation, had the strongest correlations with the drawing force caused by the roller leveler, the roller gap values and the degree of plastic deformation. The strong correlations between the steel strip properties and the operational parameters of the leveler suggest that the characteristics of the vibration signal could be predicted based on the strip properties solely. This implies that the steel strip properties could be used as input variables in models, which predict different effects on the vibration. These models could then be used in the preventive maintenance of the steel leveler.

\section{Acknowledgements}

The authors thank the personnel of SSAB Europe for collaboration and enabling of the measurement campaign during the SIMP (System Integrated Metals Processing) program coordinated by FIMECC (Finnish Metals and Engineering Competence Cluster).

\section{References}

P.S. Bullen. Handbook of Means and Their Inequalities, 2nd ed. Kluwer Academic Publishers. 2003.

Konsta Karioja, Riku-Pekka Nikula, and Toni Liedes. Vibration Measurements and Signal Processing in Stress Monitoring of a Steel Leveller. Condition Monitoring and Diagnostics \& Maintenance Performance Measurement and Management: MCMD 2015 and MPMM 2015, Oulu, Finland, 2015.

Sulo Lahdelma. On the Derivative of Real Number Order and Its Application to Condition Monitoring. Kunnossapito, 17:39-42, 1997.

Sulo Lahdelma and Esko K. Juuso. Signal Processing in Vibration Analysis. The Fifth International Conference on Condition Monitoring and Machinery Failure Prevention Technologies, Edinburgh, p. 879-889, 2008.

Sulo Lahdelma and Esko Juuso. Signal Processing and Feature Extraction by using Real Order Derivatives and Generalised Norms. Part 2: Applications. International Journal of Condition Monitoring, 1(2):54-66, 2011. doi: $10.1784 / 204764211798303814$.

Sulo Lahdelma and Vesa Kotila. Complex Derivative - A New Signal Processing Method. Kunnossapito, 19:39-46, 2005.

Riku-Pekka Nikula, Konsta Karioja, Kauko Leiviskä, and Esko Juuso. Prediction of Mechanical Stress in Roller Leveler Based on Vibration Measurements and Steel Strip Properties. Journal of Intelligent Manufacturing, 2017. doi: 10.1007/s10845-017-1341-3.

Kee-cheol Park and Sang-Moo Hwang. Development of a Finite Element Analysis Program for Roller Leveling and Application for Removing Blanking Bow Defects of Thin Steel Sheet. ISIJ International, 42(9):990-999, 2002. doi: 10.2355/isijinternational.42.990.

Richard P. Smith, Jr. Flatness Control in Coiled Plates: Lukens' Wide, Cut to Length Line. Iron and Steel Engineer, 74:29-34, 1997. 